\title{
Uma proposta do tipo ponto-proximal para o problema de quase-equilíbrio
}

\author{
Pedro Jorge S. Santos ${ }^{1}$ \\ UFPI/CMRV - UFDPar, Parnaíba, PI \\ João Carlos O. Souza ${ }^{2}$ \\ UFPI/DM, Teresina, PI
}

Resumo. Neste trabalho, estendemos os métodos propostos por Moudafi [J. Nat. Geom 15:91100, 1999] e Iusem e Sosa [Optimization 52:301-316, 2003] para o cenário de problemas de quaseequilíbrio. Analisamos as propriedades do método do tipo ponto-proximal proposto e provamos, sob hipóteses usuais, a convergência do método para uma solução do problema. Resultados numéricos preliminares são reportados.

Palavras-chave. Problema de quase-equilíbrio, Método ponto-proximal, bifunção monótona

\section{Introdução}

Seja $X \subset \mathbb{R}^{n}$ convexo, fechado e não vazio e $f: X \times X \rightarrow \mathbb{R}$ uma bifunção de equilíbrio, i.e., $f(x, x)=0$ para todo $x \in X$. O problema de equilíbrio, denotado por $\operatorname{EP}(X, f)$, consiste em encontrar $x^{*} \in X$ tal que

$$
f\left(x^{*}, y\right) \geq 0 \quad \forall y \in X .
$$

Um problema relacionado com $\operatorname{EP}(X, f)$ refere-se a encontrar $y^{*} \in X$ tal que $f\left(x, y^{*}\right) \leq 0 \forall x \in$ $X$. Este problema é conhecido como o problema dual de $\operatorname{EP}(X, f)$. Denotaremos o conjunto solução do problema de equilíbrio e de seu dual por $S_{E P}(X, f)$ e $S_{E P}^{d}(X, f)$, respectivamente.

Problemas tais como otimização, desigualdades variacionais, complementaridade, pontos fixos, pontos de sela, otimização vetorial e jogos não cooperativos, dentre outros, podem ser modelados no formato geral de problema de equilíbrio (1); veja por exemplo Blum e Oettli [4] e Santos [11].

O interesse no estudo do problema de equilíbrio nos últimos anos deve-se em grande parte ao fato de que métodos numéricos desenvolvidos para instâncias particulares de EP podem frequentemente ser estendidos para outros modelos matemáticos através da análise unificada fornecida pela formulação geral (1).

Uma clássica estratégia para resolver EP são os chamados métodos de regularização. O método de regularização de Tikhonov é bem conhecido para resolver problemas de minimização, de inclusão monótona e problemas de ponto-fixo. Esta abordagem foi estendida para resolver EP por Moudafi [10]. Este método resolve em cada iteração o problema regularizado $\operatorname{EP}\left(X, f_{k}\right)$, onde $f_{k}$ é dada por

$$
f_{k}(x, y)=f(x, y)+\gamma_{k}\left\langle x-x^{k}, y-x\right\rangle,
$$

e $\left\{\gamma_{k}\right\}$ é uma sequência auxiliar positiva e limitada. Recentemente, métodos de regularização tem sido amplamente estudados em diferentes contextos, veja por exemplo $[1,5,9]$ e suas referências.

\footnotetext{
${ }^{1}$ pedrojorge@ufpi.edu.br

2 joaocos.mat@ufpi.edu.br
} 
O objetivo deste trabalho é propor uma extensão para o problema de quase-equilíbrio de um método de regularização dado para EP. O problema de quase-equilíbrio (QEP) consiste em encon$\operatorname{trar} x^{*} \in C\left(x^{*}\right)$ tal que

$$
f\left(x^{*}, y\right) \geq 0 \quad \forall y \in C\left(x^{*}\right),
$$

onde $C: X \rightrightarrows X$ é uma aplicação ponto-conjunto de $X$ em $X$. Observe que, quando $C(x) \equiv X$ para todo $x \in X$, o problema de quase-equilíbrio retorna ao problema de equilíbrio (1). Por outro lado, é bem conhecido que problemas tais como desigualdades quase-variacionais, jogos de Nash generalizados e outros, não se encaixam na formatação de EP (1) e sim na formatação (3) de quase-equilíbrio. Veja por exemplo, $[2,11]$ e suas referências.

O problema de quase-equilíbrio possui aplicações em diversas áreas da ciência tais como engenharias, economia e pesquisa operacional; veja por exemplo [2,6] e suas referências. Algoritmos numéricos para resolver o problema de quase-equilíbrio baseados em métodos extra-gradiente, método de Newton e técnicas de funções gap têm sido propostos em [3,11,13,15,16]. Pelo que temos conhecimento nenhuma proposta do tipo ponto-proximal tem sido considerada.

Este trabalho esta organizado do seguinte modo: Na Seção 2 apresentaremos resultados preliminares relativos a EP e QEP bem como as hipóteses utilizadas. Na Seção 3 apresentaremos o algoritmo proposto e analisaremos suas propriedades. Por fim, na Seção 4 realizamos alguns experimentos numéricos básicos. Finalizamos com algumas conclusões na Seção 5.

\section{Preliminares}

Ao longo deste trabalho assumiremos as seguintes hipóteses amplamente adotadas para problemas de equilíbrio em se tratando de métodos do tipo ponto-proximal:

(P1) $f(x, x)=0$ para todo $x \in X$;

(P2) $f(\cdot, \cdot): X \times X \rightarrow \mathbb{R}$ é contínua em $X \times X$;

(P3) $f(x, \cdot): X \rightarrow \mathbb{R}$ é convexa para todo $x \in X$;

(P4) $f(x, y)+f(y, x) \leq 0$ para todo $x, y \in X ;(f$ é uma bifunção monótona $)$

(P5) Para qualquer sequência $\left\{z^{n}\right\} \subset X \operatorname{com} \lim _{n \rightarrow \infty}\left\|z^{n}\right\|=\infty$, existe $u \in X$ e $n_{0} \in \mathbb{N}$ tais que $f\left(z^{n}, u\right) \leq 0$ para todo $n \geq n_{0}$.

Os resultados a seguir foram estabelecidos para problemas de equilíbrio e desempenharão um papel importante em nossa análise de convergência. Para detalhes da prova destes resultados, veja [9, Proposition 1] e [8, Theorem 4.3].

Proposição 2.1. (a) Sob as hipóteses $(P 1-P 4)$, tem-se que $S_{E P}(X, f)=S_{E P}^{d}(X, f)$;

(b) Se, além das hipóteses $(P 1-P 4)$, supormos válida a hipótese $(P 5)$ então $S_{E P}(X, f) \neq \emptyset$.

Consideremos o problema de quase-equilíbrio consistindo em encontrar $x^{*} \in C\left(x^{*}\right)$ tal que

$$
f\left(x^{*}, y\right) \geq 0 \quad \forall y \in C\left(x^{*}\right)
$$

onde $C: X \rightrightarrows X$ é uma aplicação ponto-conjunto contínua (veja Hogan [7]) tal que para todo $x \in X, C(x) \subset X$ é não vazio, convexo e fechado. Tais hipóteses sobre a aplicação $C$ podem ser consideradas padrão no desenvolvimento de algoritmos para QEP, veja por exemplo $[3,15,16]$. 
Denotaremos por $S_{Q E P}(C, f)$ o conjunto solução do problema de quase-equilíbrio associado à bifunção $f$ e à aplicação ponto-conjunto $C$. Bem como os trabalhos de Strodiot et.al. [15] e Zhang et.al. [17], assumiremos a não vacuidade do subconjunto $S^{*} \subset S_{Q E P}(C, f)$ dado por

$$
S^{*}=\left\{x \in \bigcap_{z \in X} C(z) \mid f(x, y) \geq 0, \quad \forall y \in \bigcup_{z \in X} C(z)\right\}
$$

Dado $\gamma>0$ e $\bar{x} \in X$ fixo, considere a bifunção $\hat{f}$ dada por

$$
\hat{f}(x, y)=f(x, y)+\gamma\langle x-\bar{x}, y-x\rangle .
$$

No desenvolvimento de nosso método, utilizaremos a versão abaixo adaptada para problemas de quase-equilíbrio de resultado semelhante estabelecido em métodos de regularização para EP.

Proposição 2.2. Seja $\bar{x} \in X$ arbitrário e $\hat{x}, x^{*} \in X$ tais que $\hat{x} \in S_{E P}(\hat{f}, C(\bar{x}))$ e $x^{*} \in S_{E P}^{d}(f, C(\bar{x}))$, onde $\hat{f}$ é a bifunção dada em (6). Se f satisfaz $(P 1-P 3)$, então $\left\|\hat{x}-x^{*}\right\|^{2}+\|\bar{x}-\hat{x}\|^{2} \leq\left\|\bar{x}-x^{*}\right\|^{2}$.

Demonstração. Segue de Iusem e Sosa [9, Proposition 4] tomando $K=C(\bar{x})$ e levando em conta que $\bar{x}$ está fixado.

\section{O método}

Utilizando os resultados e notações da seção precedente, estamos prontos para estabelecer nosso algoritmo do tipo ponto-proximal para o problema de quase-equilíbrio. Analisaremos sua convergência assumindo que $f$ satisfaz as hipóteses $(P 1-P 5)$.

Algoritmo MPP: Método ponto-proximal para problemas de quase-equilíbrio

Passo 1: Tome uma sequência auxiliar limitada de parâmetros positivos $\left\{\gamma_{k}\right\}$ e escolha $x^{0} \in X$.

Passo 2: Dado $x^{k}$, calcule

$$
x^{k+1} \in S_{E P}\left(C_{k}, f_{k}\right),
$$

onde $f_{k}(x, y)=f(x, y)+\gamma_{k}\left\langle x-x^{k}, y-x\right\rangle$ e $C_{k}=C\left(x^{k}\right)$.

Passo 3: Se $x^{k+1}=x^{k}$, pare e retorne $x^{k}$. Caso contrário, ponha $k=k+1$ e volte ao Passo 2.

\subsection{A boa definição do Algoritmo MPP}

Note que a bifunção $f_{k}$ dada no Passo 2 satisfaz as hipóteses $(P 1-P 3)$, uma vez que a bifunção $f$ as satisfaz. Por outro lado, $f_{k}(x, y)+f_{k}(y, x) \leq f(x, y)+f(y, x) \leq 0$, isto é, $f_{k}$ é monótona $(P 4)$. Finalmente, Iusem e Sosa $\left[9\right.$, Proposition 3] provaram que $f_{k}$ satisfaz (P5) para todo $k \in \mathbb{N}$ desde que a bifunção original $f$ satisfaça. Portanto, $f_{k}$ satisfaz as hipóteses (P1-P5) para cada $k \in \mathbb{N}$ implicando, pela Proposição 2.1, a boa definição do Algoritmo MPP, isto é, $S_{E P}\left(C_{k}, f_{k}\right) \neq \emptyset$ para todo $k \in \mathbb{N}$ 


\subsection{Análise de convergência do Algoritmo MPP}

Iniciaremos a análise do método proposto verificando que, se o Algoritmo MPP para em algum ponto, então este ponto é solução do problema de quase-equilíbrio.

Teorema 3.1. Se a sequência gerada pelo Algoritmo MPP termina em $x^{k+1}$, então $x^{k}$ é uma solução do problema de quase-equilíbrio $Q E P(C, f)$.

Demonstração. Note que, uma vez que $x^{k+1} \in S_{E P}\left(f_{k}, C_{k}\right)$, temos que $x^{k+1} \in C\left(x^{k}\right)$ e

$$
f_{k}\left(x^{k+1}, y\right)=f\left(x^{k+1}, y\right)+\gamma_{k}\left\langle x^{k+1}-x^{k}, y-x^{k+1}\right\rangle \geq 0, \quad \forall y \in C\left(x^{k}\right) .
$$

Por outro lado, do fato de $x^{k+1}=x^{k}$ e levando em conta (8), concluimos que $x^{k} \in C\left(x^{k}\right)$ e $f\left(x^{k}, y\right) \geq 0$, para todo $y \in C\left(x^{k}\right)$. Isto é, $x^{k} \in S_{Q E P}(C, f)$.

De agora em diante, suponha que o Algoritmo MPP gera uma sequência infinita $\left\{x^{k}\right\}$.

Proposição 3.1. A sequência $\left\{x^{k}\right\}$ gerada pelo Algoritmo MPP é limitada e $\lim _{k \rightarrow \infty}\left\|x^{k+1}-x^{k}\right\|=0$.

Demonstração. Considere $x^{*} \in S^{*}$. De (5) temos que $x^{*} \in S_{E P}\left(C_{k}, f\right)$ para todo $k \in \mathbb{N}$. Por outro lado, a Proposição 2.1 garante que $x^{*} \in S_{E P}^{d}\left(C_{k}, f\right)$ para todo $k \in \mathbb{N}$. Levando em conta que $x^{k+1} \in S_{E P}\left(C_{k}, f_{k}\right)$ (conforme o Passo 2 do Algoritmo MPP) e aplicando a Proposição 2.2 com $\hat{f}=f_{k}, \bar{x}=x^{k}$ e $\hat{x}=x^{k+1}$, obtemos

$$
\left\|x^{k+1}-x^{*}\right\|^{2}+\left\|x^{k+1}-x^{k}\right\|^{2} \leq\left\|x^{k}-x^{*}\right\|^{2} \quad \forall k \in \mathbb{N} .
$$

A desigualdade acima implica que $\left\|x^{k+1}-x^{*}\right\| \leq\left\|x^{k}-x^{*}\right\|$, para todo $k \in \mathbb{N}$, o que prova a limitação da sequência $\left\{x^{k}\right\}$.

Observe que a sequência $\left\{\left\|x^{k}-x^{*}\right\|\right\}$ é monótona não crescente, e portanto, convergente. A desigualdade (9) implica em $0 \leq\left\|x^{k+1}-x^{k}\right\|^{2} \leq\left\|x^{k}-x^{*}\right\|^{2}-\left\|x^{k+1}-x^{*}\right\|^{2}$ para todo $k \in \mathbb{N}$, assim $\left\|x^{k+1}-x^{k}\right\| \rightarrow 0$ quando $k \rightarrow \infty$ um vez que $\left\{\left\|x^{k}-x^{*}\right\|\right\}$ é convergente.

Finalizamos esta seção provando que todo ponto de acumulação da sequência $\left\{x^{k}\right\}$ gerada pelo Algoritmo MPP é solução do problema de quase-equilíbrio.

Teorema 3.2. Todo ponto de acumulação da sequência $\left\{x^{k}\right\}$ pertence ao conjunto $S_{Q E P}(C, f)$.

Demonstração. Considere $\left\{x^{k_{j}}\right\}$ uma subsequência de $\left\{x^{k}\right\}$ convergindo para $\hat{x}$. Da definição do algoritmo, segue que $x^{k_{j}+1} \in C\left(x^{k_{j}}\right)$. Proposição 3.1 garante que $\left\{x^{k_{j}+1}\right\}$ converge para $\hat{x}$, e assim, da continuidade de $C$, ganhamos que $\hat{x} \in C(\hat{x})$.

Agora, dado $y \in C(\hat{x})$ qualquer, da continuidade da aplicação ponto-conjunto $C$, existe uma sequência $\left\{y^{k_{j}}\right\}$ tal que $y^{k_{j}} \rightarrow y$ e $y^{k_{j}} \in C\left(x^{k_{j}}\right)$. De $(7)$, temos que $0 \leq f\left(x^{k_{j}+1}, z\right)+\gamma_{k_{j}}\left\langle x^{k_{j}+1}-\right.$ $\left.x^{k_{j}}, z-x^{k_{j}+1}\right\rangle$, para todo $z \in C\left(x^{k_{j}}\right)$ e, em particular, para $z=y^{k_{j}}$, implica $0 \leq f\left(x^{k_{j}+1}, y^{k_{j}}\right)+$ $\gamma_{k_{j}}\left\langle x^{k_{j}+1}-x^{k_{j}}, y^{k_{j}}-x^{k_{j}+1}\right\rangle$, para todo $j \in \mathbb{N}$. Aplicando a desigualdade de Cauchy-Schwartz nesta última desigualdade, ganhamos

$$
0 \leq f\left(x^{k_{j}+1}, y^{k_{j}}\right)+\gamma_{k_{j}}\left\|x^{k_{j}+1}-x^{k_{j}}\right\| \cdot\left\|y^{k_{j}}-x^{k_{j}+1}\right\|, \quad \forall j \in \mathbb{N} .
$$

Levando em conta a Proposição 3.1, o fato de que $\left\{\gamma_{k}\right\}$ é limitada e $f$ é bifunção contínua, passando o limite na desigualdade acima com $j \rightarrow \infty$, ganhamos que $f(\hat{x}, y) \geq 0$, para todo $y \in C(\hat{x})$, i.e., $\hat{x} \in S_{Q E P}(f, C)$. 


\section{Experimentos numéricos}

Nesta seção analisaremos a performance do método proposto através de um problema acadêmico de teste em dimensão 5 proposto por Strodiot et.al. [15]. Para resolver os subproblemas do Passo 2 do Algoritmo MPP utilizamos dois algoritmos distintos encontrados na literatura de EP com a finalidade de realizar comparações. Os algoritmos utilizados foram:

- O Inexact Projected Subgradient Method (IPSM) apresentado por Santos e Scheimberg [14];

- O Newton-type Method (NM) apresentado por Santos et. al. [12].

As implementações foram feitas em MATLAB R2017b e executadas em computador pessoal Intel Core i7 com 8 GB RAM. O critério de parada adotado para o Algoritmo MPP foi $\| x^{k+1}-$ $x^{k} \|<t_{\text {qep }}$ e a sequência auxiliar $\left\{\gamma_{k}\right\}$ foi tomada constante com $\gamma_{k}=1$.

Exemplo 1. [15, Exemplo 1] Considere o QEP no qual a aplicação ponto-conjunto $C$ é dada por $C(x)=\prod_{1 \leq i \leq 5} C_{i}(x)$, onde, para cada $x \in \mathbb{R}^{5}$ e cada $i$, o conjunto $C_{i}(x)$ é definido por

$$
C_{i}(x)=\left\{y_{i} \in \mathbb{R} \mid y_{i}+\sum_{1 \leq j \leq 5, j \neq i} x_{j} \geq-1\right\} .
$$

$A$ bifunção $f$ é da forma $f(x, y)=\langle P x+Q y+q, y-x\rangle$ com $P, Q$ e q dados por

$$
P=\left[\begin{array}{ccccc}
3.1 & 2 & 0 & 0 & 0 \\
2 & 3.6 & 0 & 0 & 0 \\
0 & 0 & 3.5 & 2 & 0 \\
0 & 0 & 2 & 3.3 & 0 \\
0 & 0 & 0 & 0 & 3
\end{array}\right] ; \quad Q=\left[\begin{array}{ccccc}
1.6 & 1 & 0 & 0 & 0 \\
1 & 1.6 & 0 & 0 & 0 \\
0 & 0 & 1.5 & 1 & 0 \\
0 & 0 & 1 & 1.5 & 0 \\
0 & 0 & 0 & 0 & 2
\end{array}\right] \quad \text { e } q=\left[\begin{array}{r}
1 \\
-2 \\
-1 \\
2 \\
-1
\end{array}\right]
$$

Note que este problema não é nem um problema de equilíbrio de Nash generalizado (GNEP) nem uma desigualdade quase-variacional (QVI). Para mais detalhes, veja [15] e suas referências.

Em relação às implementações dos métodos $(I P S M)$ e $(N M)$ para resolver os subproblemas destacamos: (1) A bifunção auxiliar do método $(N M)$ adotada foi $h(x, y)=\frac{1}{2} \cdot\|y-x\|^{2}$. Computamos $y^{k}$ utilizando a subrotina quadprog. O problema de programação linear interno foi resolvido utilizando a subrotina linprog. O passo $d$ foi calculado com a subrotina linsolve. O critério de parada adotado foi o mesmo sugerido em [12] com tolerância $\varepsilon=t_{o l}$. (2) Quanto ao método $(I P S M)$ adotamos os seguintes valores para os parâmetros: $\epsilon_{k}=\xi_{k}=0, \rho_{k}=3$ e $\beta_{k}=\frac{10}{3 k}$. A projeção interna foi encontrada utilizando a subrotina quadprog. O critério de parada adotado foi o mesmo sugerido em [14] com tolerância $\varepsilon=t_{o l}$.

Os pontos iniciais utilizados foram os mesmos adotados por Strodiot et.al. [15]. Nas tabelas 5 e 6 exibimos as iteradas e a solução encontrada pelo algoritmo (com 7 casas decimais) para um dos testes realizados. Uma vez que os subproblemas foram resolvidos de modo numérico, utilizamos uma medida de "precisão" $(\Upsilon)$ para a solução encontrada. Tal medida foi calculada do seguinte modo:

$$
\Upsilon=\left\|x^{*}-\underset{y \in C\left(x^{*}\right)}{\arg \min }\left\{f\left(x^{*}, y\right)+\frac{1}{2}\left\|y-x^{*}\right\|\right\}\right\|,
$$

onde $x^{*}$ é a última iterada encontrada. Tem-se que $\Upsilon=0 \Leftrightarrow x^{*} \in S_{Q E P}(C, f)$ [11, Proposição 4.2].

Nas tabelas de 1 a 4 apresentamos um resumo do número de iteradas, tempo de cpu (em segundos) e precisão, levando em conta várias tolerâncias tol e $_{p}$ e tol $_{q e p}$ e pontos iniciais. Os testes se mostram consistentes. Observamos que para $t_{o l} p=10^{-3}$ o algoritmo possui uma menor precisão, veja tabelas 1 e 2 . 
Tabela 1: Comparações utilizando IPSM e $N M$ com $t o l_{e p}=10^{-3}$ e $t_{o l} l_{q e p}=10^{-3}$.

\begin{tabular}{c||c|c|c||c|c|c}
\hline \multirow{2}{*}{ Esquema do subproblema } & \multicolumn{3}{|c||}{$x^{0}=(1,3,1,1,2)$} & \multicolumn{3}{c}{$x^{0}=(0,0,0,0,0)$} \\
\cline { 2 - 7 } & Iter.(k) & $\mathrm{cpu}(\mathrm{s})$ & precisão $(\Upsilon)$ & Iter.(k) & $\mathrm{cpu}(\mathrm{s})$ & precisão $(\Upsilon)$ \\
\hline$I P S M$ & 2 & 0.1239 & $2.6819 \mathrm{e}-04$ & 2 & 0.1700 & $3.6263 \mathrm{e}-04$ \\
$N M$ & 1 & 0.1482 & $6.4169 \mathrm{e}-04$ & 1 & 0.1165 & $7.9729 \mathrm{e}-04$ \\
\hline
\end{tabular}

Tabela 2: Comparações utilizando IPSM e $N M$ com tol $_{e p}=10^{-3}$ e tol $_{q e p}=10^{-6}$.

\begin{tabular}{c||c|c|c||c|c|c}
\hline \multirow{2}{*}{ Esquema do subproblema } & \multicolumn{3}{|c||}{$x^{0}=(1,3,1,1,2)$} & \multicolumn{3}{c}{$x^{0}=(0,0,0,0,0)$} \\
\cline { 2 - 7 } & Iter.(k) & $\mathrm{cpu}(\mathrm{s})$ & precisão $(\Upsilon)$ & Iter.(k) & $\mathrm{cpu}(\mathrm{s})$ & precisão $(\Upsilon)$ \\
\hline$I P S M$ & 2 & 0.1582 & $2.6819 \mathrm{e}-04$ & 29 & 2.5575 & $1.2797 \mathrm{e}-04$ \\
$N M$ & 1 & 0.1299 & $6.4169 \mathrm{e}-04$ & 1 & 0.1402 & $7.9729 \mathrm{e}-04$ \\
\hline
\end{tabular}

Tabela 3: Comparações utilizando IPSM e $N M$ com tol $_{e p}=10^{-6}$ e tol $_{q e p}=10^{-3}$.

\begin{tabular}{c||c|c|c||c|c|c}
\hline \multirow{2}{*}{ Esquema do subproblema } & \multicolumn{3}{c||}{$x^{0}=(1,3,1,1,2)$} & \multicolumn{3}{c}{$x^{0}=(0,0,0,0,0)$} \\
\cline { 2 - 7 } & Iter.(k) & $\mathrm{cpu}(\mathrm{s})$ & precisão $(\Upsilon)$ & Iter.(k) & $\mathrm{cpu}(\mathrm{s})$ & precisão $(\Upsilon)$ \\
\hline$I P S M$ & 1 & 0.8349 & $3.7458 \mathrm{e}-05$ & 1 & 0.7997 & $3.4641 \mathrm{e}-05$ \\
$N M$ & 1 & 0.2513 & $3.7265 \mathrm{e}-07$ & 1 & 0.2496 & $4.4734 \mathrm{e}-07$ \\
\hline
\end{tabular}

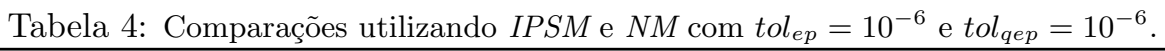

\begin{tabular}{c||c|c|c||c|c|c}
\hline \multirow{2}{*}{ Esquema do subproblema } & \multicolumn{3}{|c||}{$x^{0}=(1,3,1,1,2)$} & \multicolumn{3}{c}{$x^{0}=(0,0,0,0,0)$} \\
\cline { 2 - 7 } & Iter.(k) & cpu(s) & precisão & Iter.(k) & cpu(s) & precisão \\
\hline IPSM & 2 & 0.8332 & $6.4915 \mathrm{e}-07$ & 2 & 0.8907 & $1.8069 \mathrm{e}-07$ \\
$N M$ & 1 & 0.2503 & $3.7265 \mathrm{e}-07$ & 1 & 0.2360 & $4.4734 \mathrm{e}-07$ \\
\hline
\end{tabular}

Tabela 5: Iterações do Algoritmo MPP utilizando IPSM com tol $_{e p}=10^{-6}$ e tol $_{q e p}=10^{-6}$.

\begin{tabular}{c||c|c|c|c|c}
\hline Iter.(k) & $x_{1}^{k}$ & $x_{2}^{k}$ & $x_{3}^{k}$ & $x_{4}^{k}$ & $x_{5}^{k}$ \\
\hline 0 & 0 & 0 & 0 & 0 & 0 \\
1 & -0.7254090 & 0.8031276 & 0.7200182 & -0.8666851 & 0.2000000 \\
2 & -0.7253886 & 0.8031088 & 0.7200001 & -0.8666666 & 0.2000000 \\
\hline
\end{tabular}

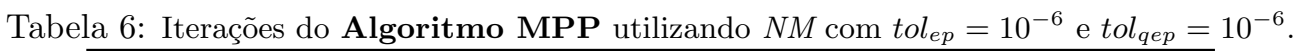

\begin{tabular}{c||c|c|c|c|c}
\hline Iter.(k) & $x_{1}^{k}$ & $x_{2}^{k}$ & $x_{3}^{k}$ & $x_{4}^{k}$ & $x_{5}^{k}$ \\
\hline 0 & 0 & 0 & 0 & 0 & 0 \\
1 & -0.7253884 & 0.8031086 & 0.7199997 & -0.8666664 & 0.2000000 \\
\hline
\end{tabular}

\section{Conclusões}

Neste trabalho propomos um método do tipo ponto-proximal para resolver problemas de quaseequilíbrio monótonos em espaços de dimensão finita. Analisamos as propriedades de convergência do algoritmo proposto e realizamos algumas experiências numéricas preliminares. As dificuldades inerentes ao problema envolvem a necessidade de se resolver simultaneamente um problema de equilíbrio e um ponto-fixo de aplicação ponto-conjunto. Os resultados encontrados até o momento se mostraram promissores. 


\section{Referências}

[1] Bento, G., Cruz Neto, J. X., Lopes, J. O., Soares Jr, P. A., Soubeyran, A. Generalized proximal distances for bilevel equilibrium problems. SIAM Journal on Optimization, 26(1):810-830, 2016.

[2] Bigi, G., Castellani, M., Pappalardo, M. and Passacantando, M. Nonlinear Programming Techniques for Equilibria. In EURO Advanced Tutorials on Operational Research. Springer, 2019. ISSN: 2364-687X.

[3] Bigi, G., Passacantando, M. Gap functions for quasi-equilibria. Journal of Global Optimization, 66(4):791-810, 2016.

[4] Blum, E., Oettli, W. From optimization and variational inequalities to equilibrium problems. The Mathematics Student, 63(1-4):123-145, 1994.

[5] Cruz Neto, J. X., Santos, P., Silva, R. C. M., Souza, J. C. O. On a Bregman regularized proximal point method for solving equilibrium problems. Optimization Letters, 13(5):1143$1155,2019$.

[6] Facchinei, F., Kanzow, C. Generalized Nash equilibrium problems. Annals of Operations Research, 175(1):177-211, 2010.

[7] Hogan, W. Point-to-set maps in mathematical programming. SIAM Review. 15:591-603, 1973.

[8] Iusem, A., Kassay, G., Sosa, W. On certain conditions for the existence of solutions of equilibrium problems. Mathematical Programming, 116(1):259-273, 2009.

[9] Iusem, A., Sosa, W. On the proximal point method for equilibrium problems in Hilbert spaces. Optimization, 59:1259-1274, 2010.

[10] Moudafi, A. Proximal point algorithm extended to equilibrium problems. Journal of Natural Geometry, 15(1-2):91-100, 1999.

[11] Santos, P. J. S., O problema de quase-equilíbrio: Uma abordagem do tipo-Newton regularizado, Tese de Doutorado, UFRJ, 2018.

[12] Santos, P. J. S., Santos, P. S. M., Scheimberg, S. A proximal Newton-type method for equilibrium problems. Optimization Letters, 12(5):997-1009, 2018.

[13] Santos, P. J. S., Santos, P. S. M., Scheimberg, S. A Newton-type method for quasi-equilibrium problems and applications. Preprint, 1-24, 2019.

[14] Santos, P. S. M., Scheimberg, S. An inexact subgradient algorithm for equilibrium problems. Computational \& Applied Mathematics, 30:91 - 107, 2011.

[15] Strodiot, J. J., Nguyen, T. T. V., Nguyen, V. H. A new class of hybrid extragradient algorithms for solving quasi-equilibrium problems. Journal of Global Optimization, 56(2):373-397, 2013.

[16] Van, N. T. T., Strodiot, J. J., Nguyen, V. H., Vuong, P. T. An extragradient-type method for solving nonmonotone quasi-equilibrium problems. Optimization, 67(5):651-664, 2018.

[17] Zhang, J., Qu, B., Xiu, N. Some projection-like methods for the generalized Nash equilibria. Computational Optimization and Applications, 45(1):89-109, 2010. 